\title{
FLAME RESPONSE TO HIGH-FREQUENCY OSCILLATIONS IN A CRYOGENIC OXYGEN/HYDROGEN ROCKET COMBUSTOR
}

\author{
N. Fdida ${ }^{1}$, J. Hardi ${ }^{2}$, H. Kawashima ${ }^{3}$, B. Knapp ${ }^{2}$, \\ M. Oschwald ${ }^{2}$, and A. Ristori ${ }^{1}$ \\ ${ }^{1}$ ONERA — The French Aerospace Lab. \\ Palaiseau 91761, France \\ ${ }^{2}$ DLR, German Aerospace Center \\ Lampoldshausen 74239, Germany \\ ${ }^{3}$ JAXA, Japan Aerospace Exploration Agency \\ Tsukuba 305-8505, Japan
}

\begin{abstract}
Experiments presented in this paper were conducted with the $\mathrm{BKH}$ rocket combustor at the European Research and Technology Test Facility P8, located at DLR Lampoldshausen. This combustor is dedicated to study the effects of high magnitude instabilities on oxygen/hydrogen flames, created by forcing high-frequency (HF) acoustic resonance of the combustion chamber. This work addresses the need for highly temporally and spatially resolved visualization data, in operating conditions representative of real rocket engines, to better understand the flame response to high amplitude acoustic oscillations. By combining ONERA and DLR materials and techniques, the optical setup of this experiment has been improved to enhance the existing database with more highly resolved $\mathrm{OH}^{*}$ imaging to allow detailed response analysis of the flame. $\mathrm{OH}^{*}$ imaging is complemented with simultaneous visible imaging and compared to each other here for their ability to capture flame dynamics.
\end{abstract}

\section{INTRODUCTION}

Combustion instabilities appeared in rocket engines along with their invention in the 1930s. High-frequency instabilities, coupling combustion and acoustics, are the most dangerous for rocket engines $[1,2]$ because they can reach amplitudes greater than $20 \%$ of the mean combustion chamber pressure and can lead to the destruction of the combustion chamber and, thus, the loss of the mission. The

(C) The Authors, published by EDP Sciences. This is an open access article distributed under the terms of the Creative Commons Attribution License 4.0 (http://creativecommons.org/licenses/by/4.0/). 
Apollo program is probably the best known for efforts to ensure the stability of the F1 motor $[3,4]$. The European Ariane program has not escaped from this phenomenon with the loss of a Viking engine during the takeoff of the second flight and the loss of the launcher $104 \mathrm{~s}$ later.

Since then, the European Community has tackled this complex issue through Franco-German research groups, such as the current program REST (Rocket Engine Stability iniTiative) to accompany the development of the Vulcain engine. The program includes theoretical studies, computational fluid dynamics (CFD) simulations, as well as experimental work to focus on different phenomena and also provide validation data for CFD codes. Among the activities, it is important to study the response of the flame to acoustic waves. That was the purpose of this study, in the context of the MOTAR ("Measurement and Observation Techniques for Aerospace Research") cooperation, to promote partnership on optical diagnostics between DLR and ONERA.

ONERA and DLR both operate research combustors to study the behavior of liquid oxygen $(\mathrm{LOx}) /$ hydrogen $\left(\mathrm{H}_{2}\right)$ rocket flames under a forced $\mathrm{HF}$ acoustic environment, by means of optical diagnostics. The ONERA multiinjector combustor (MIC) [5,6] and the DLR combustor model ' $\mathrm{H}$ ' $(\mathrm{BKH})[7,8]$ both have a rectangular combustion chamber and multiple exhaust nozzles. Under hot-fire conditions, modulating the exhaust nozzle flow rate using a toothed wheel, or siren, has proven to be an effective means of exciting transverse acoustic resonance modes of the combustion chamber. Excitation of the first transverse (1T) mode of the combustion chamber was used to study the influence of a transverse acoustic field on the injection and the flame. The transverse mode is the most common and destructive mode, if instability is encountered in a real engine. Under certain conditions, they can be self-sustaining by efficiently transferring energy from the reacting propellants to the acoustic field, quickly allowing growth to very high amplitudes which may reach the mechanical limits of the combustion chamber $[1,2]$.

Both $\mathrm{BKH}$ and MIC use large windows adapted to perform optical diagnostics in operating conditions representative of real rocket engines, that is, high propellant flowrates and high-pressure in the combustion chamber. Richecoeur et al. [9] on the MIC and Hardi et al. on the BKH [8] both observed flame response to the forced acoustic field by recording the hydroxyl-radical $\left(\mathrm{OH}^{*}\right)$ signal by means of high-speed cameras. The dynamic response of the flame submitted to the transverse HF acoustic field can only be obtained with high-speed diagnostics because the transverse collective displacement of the flame occurs with the same HF content as the acoustic disturbance.

In BKH, Webster et al. [10] showed that a nonlinear coupling was observed between measured dynamic pressure and flame response visualized with both visible and $\mathrm{OH}^{*}$ chemiluminescence imaging. Areas of high combustion rate and density gradients can be detected by $\mathrm{OH}^{*}$ emission and visible imaging, respectively. Recent work has highlighted the limitations of $\mathrm{OH}^{*}$ imaging in 
high-pressure oxygen-hydrogen combustion [11]. There are rising doubts as to its often assumed analogy with local heat release rate. The dynamic heat release rate from the flame is of relevance to studies of combustion instabilities. In past work, including that with the aforementioned MIC and $\mathrm{BKH}$ experiments, $\mathrm{OH}^{*}$ signal intensity is often taken as an indirect indicator of local heat release rate. Recent work from the Technical University of Munich has highlighted the limitations of using $\mathrm{OH}^{*}$ imaging for this purpose. In high-pressure oxygenhydrogen rocket flames, the $\mathrm{OH}^{*}$ radiation comes predominantly from thermally excited $\mathrm{OH}^{*}$ rather than chemiluminescence and, therefore, does not spatially correlate with heat release from reaction [11-13]. Furthermore, $\mathrm{OH}^{*}$ radiation suffers from strong self-absorption; so, a line-of-sight measurement images the nearest surface of the flame and is not proportional to the integrated volumetric heat release rate [14].

As a possible alternative to $\mathrm{OH}^{*}$ measurements, Webster et al. [10] applied high-speed imaging of the visible spectrum in $\mathrm{BKH}$. The results were of interest as they capture aspects of the dense flow from LOx jets as well as emission from combustion, producing a kind of 'hybrid' image. The potential of visible imaging to capture important flame dynamics and provide complementary information to $\mathrm{OH}^{*}$ imaging should be further investigated.

The current work continues the study of the influence of acoustic excitation on the flame behavior with high-speed diagnostics. The technical objective of the collaboration was achieved by combining ONERA and DLR materials: the optical setup resolution of this experiment was improved and the existing database was extended. Regarding the previous studies $[7,8]$, significant improvements have been made to record the $\mathrm{OH}^{*}$ chemiluminescence signal with a better resolution. Indeed, by combining the ONERA high-speed camera and the DLR image intensifier, both visible and $\mathrm{OH}^{*}$ camera were synchronized at $30 \mathrm{kHz}$, the shorter exposure time for $\mathrm{OH}^{*}$ was set in the middle of the visible exposure time, improving the comparability of instantaneous images. In this paper, the improved $\mathrm{OH}^{*}$ imaging is compared and contrasted with simultaneous visible imaging for its ability to capture flame dynamics. The dynamic mode decomposition method (DMD) is used to address the main response of the flow submitted to forced acoustic resonance.

\section{MATERIALS AND METHODS}

\subsection{The BKH Combustor}

Testing was conducted using the BKH combustor at the European Research and Technology Test Facility for Cryogenic Rocket Engines, 'P8,' at DLR Lampoldshausen. The BKH combustor was designed to study the interaction between 


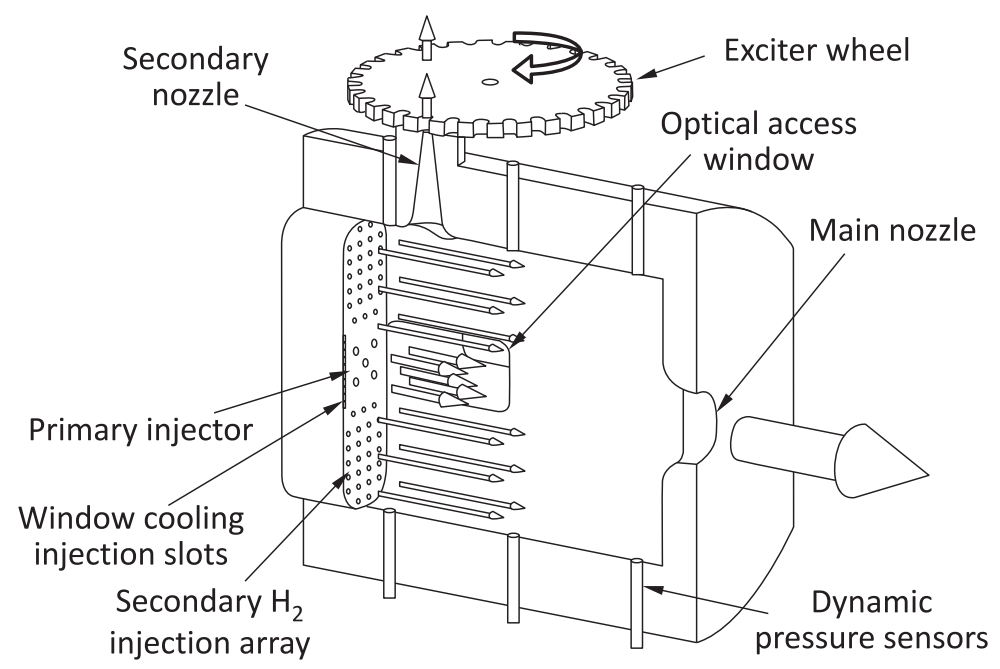

Figure 1 Illustration of the BKH combustor

$\mathrm{LOx} / \mathrm{H}_{2}$ flames and acoustics under injection conditions representative of those of an upper stage rocket engine. BKH has a rectangular cross section in order to fix the excited acoustic resonance frequencies and mode structures and optical access windows for application of high-speed imaging. A sound wave generator induces density vibrations perpendicular to the main propellant stream in the combustor. A conceptual illustration of the BKH configuration is shown in Fig. 1.

In order to shed light on the combustion behavior under acoustics, pressure and optical sensors were used to record time resolved data. BKH uses a suite of conventional, low-frequency diagnostics to specify the operating conditions, fluid temperatures, pressures, and flow rates. A set of six high-frequency Kistler pressure transducers are flush-mounted in the upper and lower combustion chamber walls. These transducers are sampled at $100 \mathrm{kHz}$ and are used for characterizing the acoustic field. Furthermore, optical windows allow access for visualization of combustion processes.

In liquid propellant rocket engines running with $\mathrm{LOx} / \mathrm{H}_{2}$, the use of shearcoaxial type elements in the injector assembly is common. The injector in BKH consists of five shear-coaxial elements arranged in a pattern which provides a representative environment for the central element, surrounded on all sides by other elements. Each element injects a central jet of dense, cryogenic LOx, and a surrounding, high-speed jet of $\mathrm{H}_{2}$. The dense 'core' of the $\mathrm{LOx}$ jet penetrates into the combustion chamber and is broken up and atomized by the shear forces exerted by the surrounding $\mathrm{H}_{2}$ flow. Three different operating conditions are 
targeted based on combustion chamber pressure: subcritical, transcritical, and supercritical, related to the critical pressure of oxygen $\left(P_{c\left(\mathrm{O}_{2}\right)}=5.04 \mathrm{~Pa}\right)$.

$\mathrm{BKH}$ has a main nozzle at the end of the combustion chamber as well as a secondary nozzle in the upper wall (see Fig. 1). The exhaust flow through the secondary nozzle is modulated with a siren to excite acoustic resonances inside the combustion chamber. The frequency of acoustic excitation is determined by controlling the rotational speed of the siren wheel. By increasing the rotational speed, the excitation frequency is ramped through a desired range between 0 and $6000 \mathrm{~Hz}$ over the course of a 40- to 70-second test firing. The $1 \mathrm{~T}$ resonance mode of the combustion chamber volume is excited as the excitation frequency passes through approximately $4200 \mathrm{~Hz}$. The structures of the acoustic pressure and velocity distributions of the $1 \mathrm{~T}$ mode were obtained from a numerical modal analysis and detailed in [8]. The $1 \mathrm{~T}$ mode has a pressure node in the near injector region which means that exciting this mode results in high amplitudes of oscillating acoustic gas motion transverse to the injection axis of the flames.

\subsection{Optical Setup}

Optical access through the $\mathrm{BKH}$ windows provides a viewing area measuring $50 \mathrm{~mm}$ high and $100 \mathrm{~mm}$ long, with one side aligned with the injection plane and the height sufficient to view the entire five-element injector. Those windows are adapted for ultraviolet (UV) and visible imaging to record simultaneously the reaction zone of the flame and the visible emission of the flame. A film cooling of hydrogen is injected along the windows to protect them from thermal shocks. The setup of high-speed cameras is illustrated schematically in Fig. 2.

The $\mathrm{OH}$ radical $\left(\mathrm{OH}^{*}\right)$ is an intermediate species of oxygen/hydrogen combustion which is produced in the reaction (flame) zone [15]. Thus $\mathrm{OH}^{*}$ chemiluminescence can be used to locate the flame front. Radiation from $\mathrm{OH}^{*}$ takes place in the near UV range between 306 and $320 \mathrm{~nm}$ [16]. $\mathrm{OH}^{*}$ emission is collected with a high-speed intensified camera placed on one side of the combustion chamber, as shown in Fig. 2. A dichroic mirror is used to split UV light to the ONERA camera for $\mathrm{OH}^{*}$ imaging and the visible light by the DLR camera.

Two high-speed cameras were used to characterize the flame behavior during acoustic excitation and off-resonance. The visible flame luminosity is recorded with a Photron Fastcam SA5. Twelve-bit images were recorded with a frame rate of $30000 \mathrm{fps}$, a shutter speed of $1.0 \mu \mathrm{s}$, and an image size of $640 \times 376$ pixels. A Sigma lens of focal $500 \mathrm{~mm}$ was mounted in front of the camera and the aperture was fixed to 5.6. The camera settings result in a resolution of $0.17 \mathrm{~mm}$ per pixel. The velocity of LOx at the point of injection is around $12 \mathrm{~m} / \mathrm{s}$, resulting in a flow displacement of far less than 1 pixel $(\sim 0.012 \mathrm{~mm})$ during exposure time. 


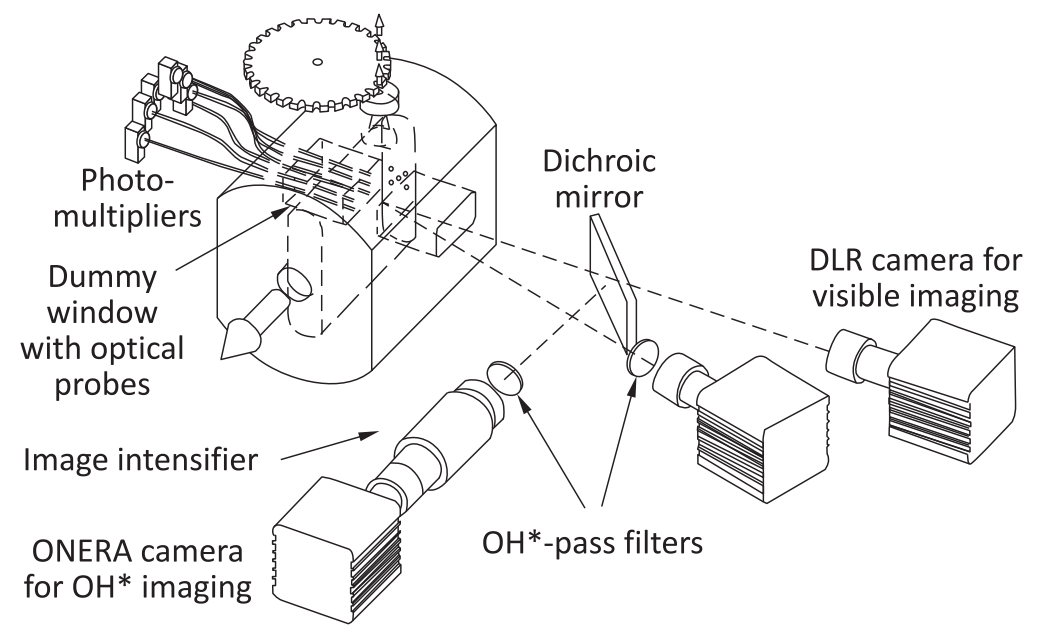

Figure 2 Optical setup on the BKH combustor

An UV dichroic mirror allows a second high-speed camera to record $\mathrm{OH}^{*}$ chemiluminescence simultaneously. This mirror acts like a beam splitter so that there is no overlap between the spectrum seen by the $\mathrm{OH}^{*}$ and visible cameras, due to the high $(>99 \%)$ reflectance of the beam splitter from 290 to $330 \mathrm{~nm}$. A Vision Research Phantom v711 was combined with a high-speed intensifier for UV light (Hamamatsu C10880). The intensifier is set with a short exposure time of $350 \mathrm{~ns}$ to freeze the $\mathrm{OH}^{*}$ signal on 12-bit images. The frame rate was set to $30000 \mathrm{fps}$ which ensures to record the dynamic flame response to the acoustic field. The image size was set to $480 \times 224$ pixels leading to a resolution of $0.23 \mathrm{~mm} /$ pixel. An UV lens of $94 \mathrm{~mm}$ of focal length and opened at f/5.6 was used to collect UV light from the flame. A band pass filter, centered on $310 \mathrm{~nm}$ with a FWHM (Full Width at Half Maximum) of $10 \mathrm{~nm}$ and a transmission peak of $15 \%$ at $310 \mathrm{~nm}$, was placed in front of the intensifier to select the $\mathrm{OH}^{*}$ chemiluminescence.

The technical objective of the collaboration was to improve the quality of $\mathrm{OH}^{*}$ imaging compared to previous studies $[7,8]$ and it was achieved by combining the ONERA high-speed camera and DLR image intensifier. The actual spatial resolution in this setup $(480 \times 224$ pixels, $0.23 \mathrm{~mm} / \mathrm{pixel})$ constitutes a factor of 1.9 improvement, showing more detailed features of the turbulent flame. The temporal resolution has also been improved by a factor of 1.2 (recording rate $30 \mathrm{kfps}$ ), which captures frequencies up to the second overtone of the $1 \mathrm{~T}$ mode. Moreover, the visible and $\mathrm{OH}^{*}$ cameras were synchronized and the shorter exposure time for $\mathrm{OH}^{*}$ (350 ns) was set in the middle of the visible exposure time of $1 \mu \mathrm{s}$, improving the comparability of instantaneous images. The dy- 
namic range of the CMOS (complementary metal oxide semiconductor) sensor is 12 bit instead of 8 bit in the previous experiment and constitutes a significant improvement as the $\mathrm{OH}^{*}$ signal was recorded on $50 \%$ of the dynamic range. All together, this setup provides a more detailed analysis of the flame. With this setup, the recording time of the cameras is nearly the same, about $1.65 \mathrm{~s}$. Both cameras are triggered at the same time, when the siren frequency is approaching the $1 \mathrm{~T}$ mode resonance.

\subsection{Operating Conditions}

BKH operates with injection parameters and mean chamber pressures $\left(P_{\mathrm{cc}}\right)$ which are representative of real, upper-stage liquid propellant rocket engines. Among the hot runs performed during this campaign, imaging results are examined from the test with chamber pressure $P_{\mathrm{cc}}=60$ bar, using liquid hydrogen $\left(\mathrm{LH}_{2}\right)$ and $\mathrm{LOx}$ as propellants and with an oxidiser-to-fuel ratio (ROF) of 6 . The total mass flow rate per injector element is $134 \mathrm{~g} / \mathrm{s}$, the hydrogen-to-oxygen velocity ratio $\mathrm{VR}=u_{\mathrm{H}} / u_{\mathrm{O}}$ is 8.6 , and the momentum flux ratio $J=\left(\rho u^{2}\right)_{\mathrm{H}} /\left(\rho u^{2}\right)_{\mathrm{O}}$ $=1.5$ where subscripts $\mathrm{O}$ and $\mathrm{H}$ refer to oxygen and hydrogen, respectively.

Figure 3 shows the $P_{\mathrm{cc}}$ and ROF signals over the course of the test run. After the 10-second startup transient phase, $P_{\mathrm{cc}}$ reaches the targeted value of 60 bar. The acoustic excitation frequency, shown as curve 3 in Fig. 3, increases over the course of the test, in this case, with a nonlinear profile. The acoustic frequency reaches the $1 \mathrm{~T}$ mode of the combustion chamber, at nearly $4.5 \mathrm{kHz}$, about $28 \mathrm{~s}$

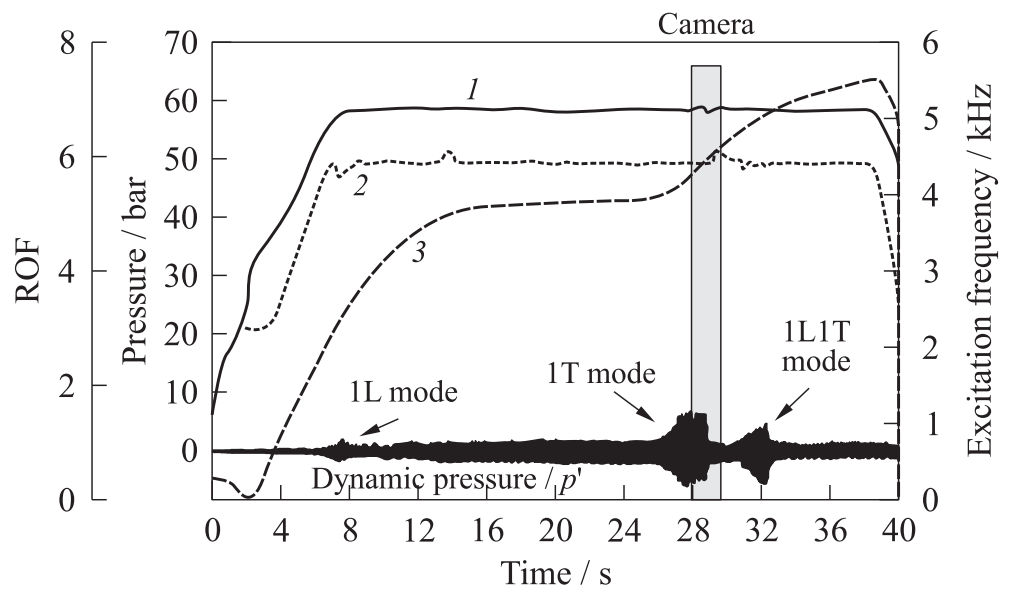

Figure 3 Test profiles (1L - first longitudinal and $1 \mathrm{~L} 1 \mathrm{~T}$ - first combined longitudinal-transverse): $1-P_{\mathrm{cc}} ; 2-\mathrm{ROF}$; and $3-$ excitation frequency 


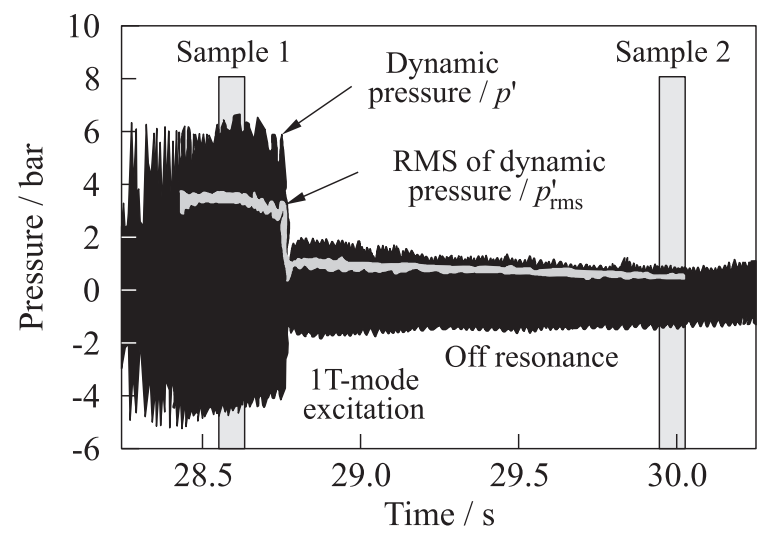

Figure 4 Closeup view of the dynamic pressure signal showing the origin of the chosen optical samples

from the start of acquisition. Cameras were triggered by the wheel frequency to get images during the $1 \mathrm{~T}$-mode resonance, with a total recording period of $1.65 \mathrm{~s}$. A closeup view of the dynamic pressure signal during 1T-mode excitation, highpass filtered to exclude combustion noise, is shown in Fig. 4. Two sampling periods for camera images are defined: Sample 1 is at the peak of 1T-mode forced resonance, as confirmed by the root mean square (RMS) of dynamic pressure overlaid in Fig. 4, and Sample 2 is considered as off-resonance, where the RMS of dynamic pressure is the lowest.

\subsection{Postprocessing}

For a qualitative comparison of the imaging techniques, time-averaged images were calculated over the 2000 instantaneous $\mathrm{OH}^{*}$ chemiluminescence and visible images comprising Samples 1 and 2. These suffice for a comparison of mean flame structure.

The study of acoustic-flame interaction has been facilitated by the application of high-speed diagnostics capable of kilohertz acquisition rates. Image acquisition at such rates can lead to substantially large data sets that are difficult to process and analyze. One powerful means of analyzing such data sets is through the use of decomposition methods such as DMD. This type of analysis can provide low-order estimates of higher order data obtained from high-speed measurements. These estimates can then serve as a representation for the entire data set, providing information that is more meaningful to the researcher for understanding turbulent-flow environments. Rowley et al. [17] and Schmid [18] provide detailed descriptions of the method. Application of such a decomposi- 
tion method to turbulent flames was performed, for example, by Richecoeur et al. [19] and Bourgouin et al. [20] to highlight dynamical couplings in reactive turbulent flows. The DMD attempts to represent a data sequence by orthogonal components in time where each mode is associated to a single frequency. In this experiment, where the acoustic excitation is imposed, the coherence of the flow can be directly linked to the acoustic frequency.

\section{RESULTS AND DISCUSSION}

\subsection{Qualitative Comparison of Imaging}

Instantaneous images from both imaging techniques are compared for the same time instant from Sample 2, referred to as the off-resonance case. The images are shown in Fig. 5, with visible above and $\mathrm{OH}^{*}$ below, and a projection of the combustor cross section for orientation purposes. For display, the images have been normalized to the same intensity scale and resized for side-by-side comparison. The visible spectrum recorded by the camera is defined by the spectral sensitivity of the CMOS sensor, which ranges from 400 to $900 \mathrm{~nm}$,

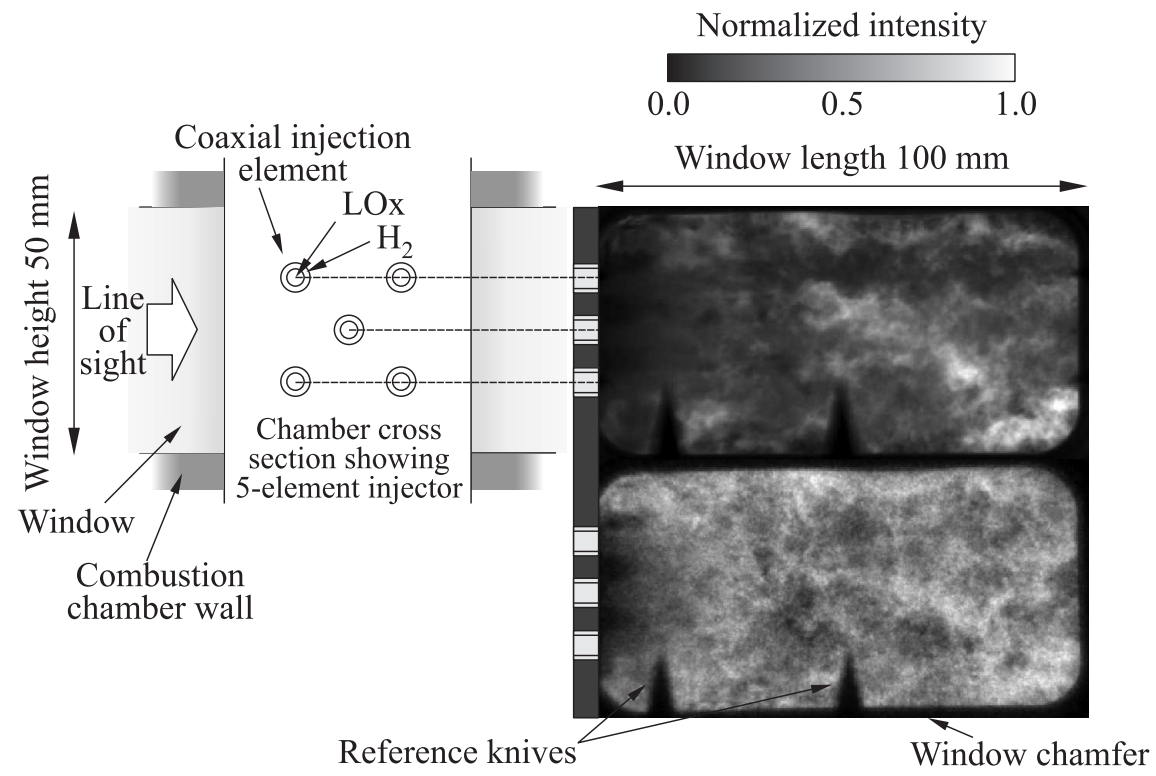

Figure 5 Instantaneous visible (above) and $\mathrm{OH}^{*}$ emission (below) images, from Sample 2 for off-resonance excitation 
peaking at around $650 \mathrm{~nm}$. This includes the characteristic blue continuum of oxygen-hydrogen flames, which peaks between 420 and $450 \mathrm{~nm}$, and will, therefore, dominate the content of the image. The $\mathrm{OH}^{*}$ emission signal, on the other hand, is given by the band-pass filter, which focuses on a narrow part of the $\mathrm{OH}$ system. Differences in intensity distribution may also be influenced by the exposure time for each image, with that for visible three times longer than for $\mathrm{OH}^{*}$. Furthermore, cameras record light from slightly different collection angles, as their focal lengths are different, with the same aperture number of 5.6.

Similarities between images from each camera can be noticed, even if the spectra of wavelengths captured by each do not overlap. Two vertical knives can be seen at the bottom of each image, which are used as spatial references and are positioned outside of the combustion chamber. A close look indicates that the knife edges in the visible imaging are more diffuse, indicating a smaller depth-of-field for visible imaging, due to the longer focal length of the lens used.

Both images show a turbulent flame which fills the window field of view, with individual flames from the five injection elements indistinguishable from each other. Some corresponding regions of intensity patterns can be identified in both images, a prominent example of which is in the lower right quarter of the window. In general, intensity gradients are stronger in the visible imaging, whereas the $\mathrm{OH}^{*}$ intensity distribution is more homogeneous. On the left side of both images, the confined structure of the flame formed by the high-speed propellant jets is discernible. In the $\mathrm{OH}^{*}$ image, these quickly expand to overlap and be lost in the turbulent field, whereas dense LOx jet structures can be discerned for much longer distances downstream in the visible image.

These features become more easily recognizable in the time-averaged images for Sample 2 in Fig. $6 a$. In particular, the LOx jets from the upper and lower injection elements can be traced nearly the length of the window in the visible image. The fact that the LOx jets can be recognized means the visible radiation is optically thin. This is consistent with the conclusions of Fiala and Sattelmayer [21], who compared measurements of $\mathrm{OH}^{*}$ and blue wavelengths in a laminar flame up to 40 bar. They showed that the dominant blue radiation from hydrogen flames does not suffer from self-absorption as does $\mathrm{OH}^{*}$ radiation. Furthermore, through modeling potential sources, they identified its origin as chemiluminescence in the formation of $\mathrm{H}_{2} \mathrm{O}_{2}$, which indicates that visible radiation correlates with the reaction zone better than $\mathrm{OH}^{*}$. In Fig. 6, the $\mathrm{LOx}$ jets can be made out for the first few millimetres after injection as a dark grey form on a black background. This means one sees visible radiation emitted from the surface of the LOx jet, in the shear layer where it is reacting with the coaxial $\mathrm{H}_{2}$ stream. Further downstream, the jets appear darker than the surrounding brighter flame. This is assumed to be the result of absorption of visible radiation from the flame in the region behind the LOx jet, so that either side of the LOx jet the integrated line-of-sight signal is more intense than that from near side of 


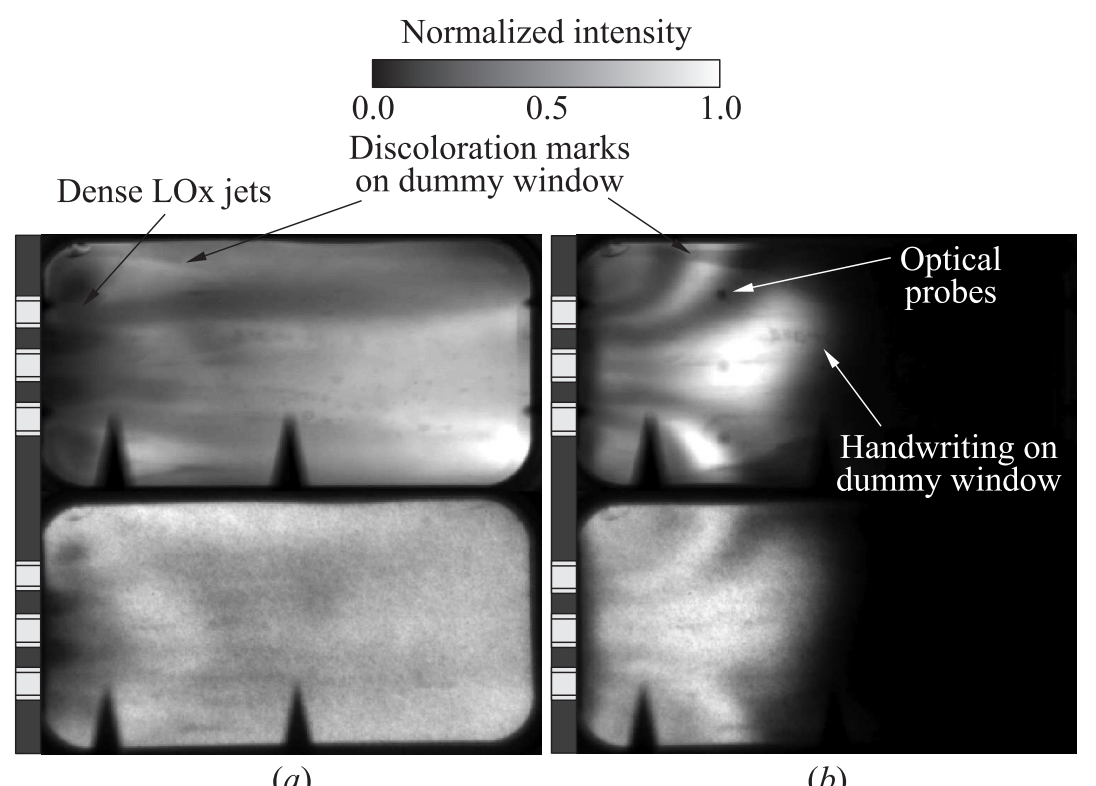

Figure 6 Time-averaged visible (above) and $\mathrm{OH}^{*}$ emission (below) images, from Sample 2 for off-resonance conditions $(a)$ and Sample 1 for 1T-mode resonance $(b)$

the LOx jet only. In the $\mathrm{OH}^{*}$ image, the recirculation zone from the upper element is bright and well defined in the upper left corner of the image, whereas it is not as prominent in the visible image. Furthermore, discoloration marks from past tests can be seen on the surface of the dummy window in the background of the image. These features again indicate a large degree of transparency of the flame in the visible spectrum, compared to the relative opacity of the $\mathrm{OH}^{*}$ field.

Figure $6 b$ shows the time-averaged images during the period of $1 \mathrm{~T}$-mode excitation (Sample 1). These show the global behavior of the flame under 1Tmode resonance, in contrast with the off-resonance case in Fig. $6 a$. The length of the flame is clearly shortened during the 1T-mode excitation, which is mainly due to the effect of acoustics on the LOx jet. Hardi et al. [7] showed a strong influence of the transverse acoustic field on the LOx jet in past work with $\mathrm{BKH}$, showing that the mechanism of the dense core breakup and atomization differs between off-resonance and 1T-mode excitation. The core length was found to decrease with increasing amplitude of acoustic pressure, with a core length reduction up to $70 \%$ under amplitudes representative of dangerous combustion instabilities. Those studies, as for the current work, were made using excitation of the $1 \mathrm{~T}$ mode, around $4200 \mathrm{~Hz}$. This is close to the $1 \mathrm{~T}$ frequency in real, upper-stage 
engines. BKH was deliberately designed with this $1 \mathrm{~T}$-mode trait so as to result in spray-acoustic interaction at representative time scales.

As for the off-resonance images, there are areas where strong $\mathrm{OH}^{*}$ signal correlates spatially with strong visible emission. The dense LOx jets are still more easily discerned in the visible image than in the $\mathrm{OH}^{*}$ image. Now, as the outer jets are deflected away from the chamber main axis, they obscure the flame from the central element to a far lesser extent. This further improves the visibility of the LOx jet from the central element, appearing as a straight, dark form framed by the high intensity flame radiation surrounding it. This again indicates low opacity of the half of the flame located between the jet and the visible camera. Furthermore, the retracted flames have allowed other features to become evident which highlight the difference in opacity of the imaged wavelength bands. In the visible image, the locations of the upstream row of optical probes in the dummy window are clearly visible and even some handwriting on the surface of the dummy window can be seen. This is not the case in the $\mathrm{OH}^{*}$ image where the LOx jets and back wall of the chamber are not discernible. In summary, this qualitative comparison of the two types of imaging in rocket flames at 60 bar, for both unperturbed and acoustically forced cases, is consistent with current understanding of the luminescence of laminar oxygen-hydrogen flames at lower pressures.

\subsection{Modal Decomposition}

Acquiring images with a high repetition rate allows resolution of the HF content of the dynamics of this reacting flow. The data can be useful in understanding the flame response and the time delay between the heat release and the acoustic disturbance [8]. Signals from pressure transducers, $\mathrm{OH}^{*}$ emission, and visible wavelengths can have similar frequency content, as shown by Webster et al. [10] in a past BKH test case. Here, it is proposed to compare the dynamic flame response by applying a DMD method on the visible and $\mathrm{OH}^{*}$ images.

Dynamic mode decomposition method was applied to the respective sets of 2000 images from Sample 1 to detect coupling between the reacting flow and acoustics during 1T-mode resonance. The mode energy spectrum obtained with DMD for both types of imaging is shown in Fig. 7, presenting modes organized by increasing frequencies. The acoustic spectrum from a dynamic pressure sensor in the combustion chamber is included for comparison. Peaks in the DMD spectrum are linked to the eigenfrequencies of the combustion chamber, when the flame luminosity is driven by acoustics. This indicates strong, coherent, periodic flow structures at the eigenfrequencies of the combustion chamber.

Both $\mathrm{OH}^{*}$ and visible imaging show a dominant response to the main excitation frequency at $4470 \mathrm{~Hz}$. The first and second harmonics of the pri- 


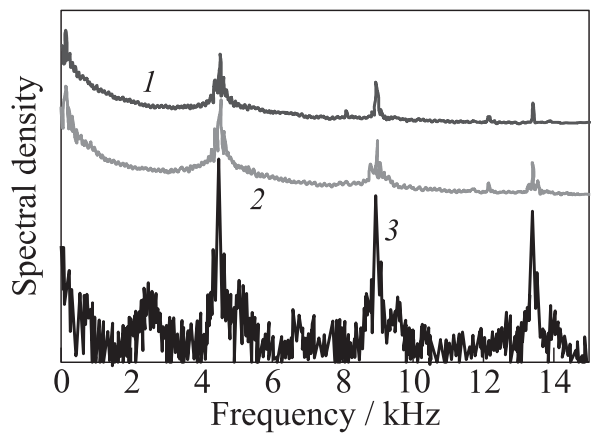

Figure 7 Frequency spectra of DMD mode energy from imaging ( $1-\mathrm{OH}^{*}$; and 2 - visible) and dynamic pressure (3) in the combustion chamber from Sample 1: 1 DMD mode energy, $\mathrm{OH}^{*}$ imaging; 2 - DMD mode energy, visible imaging; and 3 dynamic pressure

mary excitation frequency are also prominent at 8925 and $13395 \mathrm{~Hz}$, respectively. The frequencies of the spectral peaks are well defined and correspond to those in the acoustic spectrum perfectly. This definition is not affected by the ramped acoustic excitation because, over the sampling period of $66 \mathrm{~ms}$, the wheel frequency increases by about $20 \mathrm{~Hz}$ which is less than the spectral resolution.

The modes corresponding to the three peaks in the acoustic spectrum are selected for further examination. The normalized, real components of these three modes are plotted in Fig. 8, visible above and $\mathrm{OH}^{*}$ below, to compare the spatial organization of the amplitude of flame response. In the visible images, the energy amplitude varies on a gray scale linked to the spatial variation of the LOx jet and to the flame emission, which is driven by the first eigen forced frequency [8]. $\mathrm{OH}^{*}$ show similar energy distribution, linked to the heat release, to a certain extent, oriented vertically, alternatively with the acoustic velocity of the $1 \mathrm{~T}$ mode. The transverse acoustic velocity causes a transverse convective displacement of the flame simultaneously with the acoustic oscillation, as can be seen in the instantaneous $\mathrm{OH}^{*}$ images. Regions of high intensity fluctuation correlate more strongly between the visible and $\mathrm{OH}^{*}$ images on DMD modes than in the time-averaged images. Both show strong 'branch-like' regions of intensity fluctuation, with opposite phase, above and below the centerline of the LOx jet. This can be interpreted as representing oscillating vertical displacement of the flame, confirming that during this period, the heat release and the flame luminosity are driven by the transverse acoustic oscillations $[8,10]$.

The distributions of the two harmonic modes in Fig. 8 also show a strong spatial coherence, with branch structures of higher spatial order complementing those in the primary $1 \mathrm{~T}$ mode (see Fig. $8 a$ ). This shows that the flame is 


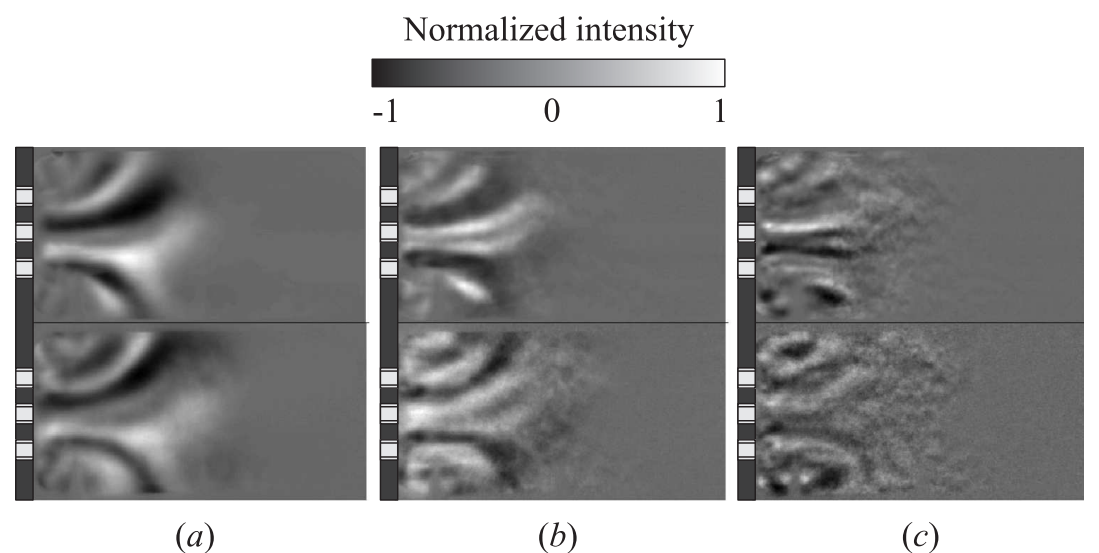

Figure 8 Real components of the three selected DMD modes, from visible (above) and $\mathrm{OH}^{*}$ imaging (below), with increasing frequency from left to right

responding nonlinearly to the nonlinear acoustic perturbation. Together, the three DMD modes shown here appear to capture the dominant flame dynamics in response to the acoustic excitation, as it is displaced vertically back and forth in unison with the acoustic particle velocity. In the first mode (see Fig. 8a), the branches either side of the centerline, with 180 degree relative phase, reflect the lobes of increased emission produced alternately above and below the LOx jet with each passing transverse wave and entrained translation of the bulk of the flame.

The second mode (see Fig. 8b) contains structures at a frequency twice as large as the spatial frequency in the vertical direction with relative phase consistent with the appearance of strongest intensity centered on each LOx jet twice per acoustic cycle.

The third mode (see Fig. $8 c$ ) is not as coherent or simple to interpret as the first two, but appears to refine the information on the turbulent recirculation zones near the faceplate. Differences between the modes from visible and $\mathrm{OH}^{*}$ imaging which are consistent with previous observations can also be noticed.

Gradients in fluctuation intensity are somewhat stronger for visible than for $\mathrm{OH}^{*}$ modes. This is particularly evident for the branch structures around the central LOx jet in the second and third modes. In the third mode especially, the branches with antiphase indicate strong activity along the upper and lower surfaces of the LOx jet which is not evident the $\mathrm{OH}^{*}$ mode. These features of flame dynamics near the LOx jet are accessible in the visible image due to the high transparency of the flame, in contrast to the $\mathrm{OH}^{*}$ image where they are obscured by the foremost surface of the flame. 
In the first mode, the visible distribution appears somewhat lifted compared to the $\mathrm{OH}^{*}$ distribution. This is consistent with the region of stronger visible emission which begins shortly downstream of injection, whereas recirculated flame of even small thickness appears brightly in $\mathrm{OH}^{*}$ emission. Similarly, the recirculation zones in the upper and lower left-hand corners of the window are strongly evident in the $\mathrm{OH}^{*}$ modes, but only weakly in the corresponding visible modes.

In comparison with these DMD results from Sample 1 during 1T-mode resonance, DMD applied on images for off-resonance excitation of Sample 2 shows no dominant spectral features and modes with very weak spatial coherence. These results are not shown here as they serve little more than do the time-averaged images in Fig. 6.

Both imaging methods have proven to be complementary in highlighting the flow features and flame dynamics in response to acoustic forcing. Due to the nature of $\mathrm{OH}^{*}$ emission and processes associated with production of $\mathrm{OH}, \mathrm{OH}^{*}$ imaging appears to be more opaque and can, therefore, be thought of as depicting the surface of the spatially oscillating flame. Visible imaging is sensitive to continuous emission of the flame and other products of combustion, which have a degree of transparency allowing the presence of the LOx core to be detected. In future work, correlation methods or image differencing between the two types could help isolate features of the flow and their response to acoustics.

\section{CONCLUDING REMARKS}

This collaborative work extends the existing DLR database of oxygen-hydrogen cryogenic flames submitted to a high-magnitude transverse acoustic field. The combination of ONERA and DLR high-speed diagnostics improved the quality of previous $\mathrm{OH}^{*}$ imaging with a better spatial and temporal resolution, as well as the dynamic range. This experimental work was the opportunity for DLR, ONERA, and JAXA to share and compare techniques in order to improve the technical standard of the visualization of cryogenic spray flame response under representative rocket conditions and forced acoustic perturbation.

High-speed films were recorded at $30 \mathrm{kHz}$ to capture the flame dynamics submitted to a transverse acoustic field at $4.5 \mathrm{kHz}$, simultaneously for the $\mathrm{OH}^{*}$ and the visible emission of the flame. The two imaging methods were qualitatively compared for samples from 1T-mode resonance and off-resonance acoustic excitation. Both methods are sensitive to emission from combustion, with the broad visible wavelength region having a high degree of transparency compared to the narrow, selective $\mathrm{OH}^{*}$ region. The $\mathrm{OH}^{*}$ imaging, therefore, has the appearance 
of the flame outer surface, whereas features of the flow field such as the dense oxygen jets are evident in the visible imaging. This finding is consistent with current knowledge of the nature of luminescence in hydrogen-oxygen flames. The origin of the radiation and its optical path must be taken into account when interpreting features in the images.

Dynamic mode decomposition was applied to study the coherence of the emission intensity field submitted to the forced acoustic field. Although both cameras do not see the same emission spectrum, DMD applied on both kinds of images reveals similar features of the flow dynamics. The $\mathrm{OH}^{*}$ emission and visible luminosity of the flame show similar temporal dynamics, with the three most energetic DMD modes at frequencies corresponding to the three peaks in the combustion chamber acoustic spectrum arising from the forcing of the transverse mode. Together, the mode distributions describe the transverse displacement of the flame driven by the transverse acoustic field. Features of this response near the dense oxygen jets are accessible in the decomposition of the visible imaging which are obscured in the $\mathrm{OH}^{*}$ imaging.

In future work, it is intended to compare the two types of imaging in more detail in order to better understand their complementary features. For example, localizing areas of maximum signal for $\mathrm{OH}^{*}$ and visible imaging could show differences indicating the fluid structure. The phase response could also be compared to show if there is a delay between the response observed in both imaging methods, in case the acoustic field affects the LOx jet and the $\mathrm{OH}^{*}$ chemiluminescence differently.

\section{ACKNOWLEDGMENTS}

This work was performed in the framework of the MOTAR agreement between ONERA and DLR. This research program is aimed at developing cooperation between ONERA and DLR in optical diagnostics, such as those applied to rocket engine combustors. The work is also associated with the Franco-German REST cooperation.

The authors are grateful to the P8 test bench team for their professional effort in test operations, as well as the DLR and ONERA optical team for setting up and operating the optical diagnostics.

Thanks to Scott Beinke, Frédéric Levy, and Matthew Wierman for their valued support in modal decomposition analysis.

\section{REFERENCES}

1. Harrje, D. T., and F. H. Reardon. 1972. Liquid propellant rocket combustion instability. NASA-SP-194. 
2. Barrère, M., and F. Williams. 2001. Comparison of combustion instabilities found in various types of combustion chambers. 12th Symposium (International) on Combustion. 169-181.

3. Yang, V., and W. Anderson, eds. 1995. Liquid rocket engine combustion instability. Progress in astronautics and aeronautics ser. Washington, D.C.: AIAA. Vol. 169. $657 \mathrm{p}$.

4. Biggs, R. 2009. F-1 Saturn V stage engine, remembering the giants. Chapter 1, F-1 Saturn V Stage Engine. Monograph in aerospace history No. 45. Apollo Rocket Propulsion Development.

5. Richecoeur, F., P. Scouflaire, S. Ducruix, and S. Candel. 2006. High frequency transverse acoustic coupling in a multiple injector cryogenic combustor. J. Propul. Power 22:790-799.

6. Méry, Y., L. Hakim, P. Scouflaire, L. Vingert, S. Ducruix, and S. Candel. 2013. Experimental investigation of cryogenic flame dynamics under transverse acoustic modulations. Comptes Rendus Mécanique 341:100-109.

7. Hardi, J. S., H. C. Martinez, M. Oschwald, and B. B. Dally. 2014. LOx jet atomization under transverse acoustic oscillations. J. Propul. Power 30(2):337-349.

8. Hardi, J. S., S. K. Beinke, M. Oschwald, and B. B. Dally. 2014. Coupling of cryogenic oxygen-hydrogen flames to longitudinal and transverse acoustic instabilities. J. Propul. Power 30(4):991-1004.

9. Richecoeur, F., S. Ducruix, P. Scouflaire, and S. Candel. 2008. Experimental investigation of high-frequency combustion instabilities in liquid rocket engine. Acta Astronaut. 62(1):18-27.

10. Webster, S. C. L., J. S. Hardi, and M. Oschwald. 2014. High pressure visualisation of liquid oxygen and cryogenic hydrogen combustion under an imposed acoustic field. 19th Australasian Fluid Mechanics Conference.

11. Fiala, T., and T. Sattelmayer. 2013. On the use of $\mathrm{OH}^{*}$ radiation as a marker for the heat release rate in high-pressure hydrogen-oxygen liquid rocket combustion. 5th EUCASS Conference.

12. Fiala, T. 2015. Radiation from high pressure hydrogen-oxygen flames and its use in assessing rocket combustion instability. Technische Universität München. PhD Thesis.

13. Fiala, T., and T. Sattelmayer. 2015. Heat release and UV-Vis radiation in nonpremixed hydrogen-oxygen flames. Exp. Fluids 56(144). doi: 10.1007/s00348-0152013-8.

14. Fiala, T., and T. Sattelmayer. 2016. Assessment of existing and new modeling strategies for the simulation of $\mathrm{OH}^{*}$ radiation in high-temperature flames. CEAS Space J. 8:47-58. doi: 10.1007/s12567-015-0107-z.

15. Gaydon, A. G. 1957. The spectroscopy of flames. Chapman \& Hall Ltd. 288 p.

16. Dieke, G.H., and H.M. Crosswhite. 1962. The ultraviolet bands of $\mathrm{OH}$ fundamental data. J. Quant. Spectrosc. Ra. Transfer 2(2):97-199. doi: 10.1016/00224073(62)90061-4.

17. Rowley, C. W., I. Mezic, S. Bagheri, P. Schlatter, and D. S. Henningson. 2009. Spectral analysis of nonlinear flows. J. Fluid Mech. 641:115-127. 
18. Schmid, P. J. 2010. Dynamic mode decomposition of numerical and experimental data. J. Fluid Mech. 656:5-28.

19. Richecoeur, F., L. Hakim, A. Renaud, and L. Zimmer. 2012. DMD algorithms for experimental data processing in combustion. Proceedings of the Summer Program 2012 of Center of Turbulence Research. 459-468.

20. Bourgouin, J.F., J. Moeck, D. Durox, T. Schuller, and S. Candel. 2013. Sensitivity of swirling flows to small changes in the swirler geometry. Comptes Rendus Mécanique 341(1):211-219.

21. Fiala, T., and T. Sattelmayer. 2014. On the origin of the continuous (blue) radiation in hydrogen flames. Sonderforschungsbereich/Transregio 40. Annual Report. 